University of Wollongong

Research Online

Faculty of Engineering and Information

Faculty of Engineering and Information

Sciences - Papers: Part B

Sciences

2018

\title{
Temporal separation of Cerenkov radiation and scintillation using artificial neural networks in Clinical LINACs
}

Levi Madden

University of Wollongong, ljm898@uowmail.edu.au

James Archer

University of Wollongong, jia335@uowmail.edu.au

Enbang Li

University of Wollongong, enbang@uow.edu.au

Dean Wilkinson

University of Wollongong, deanw@uow.edu.au

Anatoly B. Rosenfeld

University of Wollongong, anatoly@uow.edu.au

Follow this and additional works at: https://ro.uow.edu.au/eispapers1

Part of the Engineering Commons, and the Science and Technology Studies Commons

Research Online is the open access institutional repository for the University of Wollongong. For further information contact the UOW Library: research-pubs@uow.edu.au 


\title{
Temporal separation of Cerenkov radiation and scintillation using artificial neural networks in Clinical LINACs
}

\author{
Abstract \\ The irradiation of scintillator-fiber optic dosimeters by clinical LINACs results in the measurement of \\ scintillation and Cerenkov radiation. In scintillator-fiber optic dosimetry, the scintillation and Cerenkov \\ radiation responses are separated to determine the dose deposited in the scintillator volume. Artificial \\ neural networks (ANNs) were trained and applied in a novel single probe method for the temporal \\ separation of scintillation and Cerenkov radiation. Six dose profiles were measured using the ANN, with \\ the dose profiles compared to those measured using background subtraction and an ionisation chamber. \\ The average dose discrepancy of the ANN measured dose was $2.2 \%$ with respect to the ionisation \\ chamber dose and $1.2 \%$ with respect to the background subtraction measured dose, while the average \\ dose discrepancy of the background subtraction dose was $1.6 \%$ with respect to the ionisation chamber \\ dose. The ANNs performance was degraded when compared with background subtraction, arising from \\ an inaccurate model used to synthesise ANN training data.

\section{Disciplines} \\ Engineering | Science and Technology Studies

\section{Publication Details} \\ Madden, L., Archer, J., Li, E., Wilkinson, D. \& Rosenfeld, A. (2018). Temporal separation of Cerenkov \\ radiation and scintillation using artificial neural networks in Clinical LINACs. Physica Medica: an \\ international journal devoted to the applications of physics to medicine and biology, 54 131-136.
}




\begin{abstract}
Convolutional neural network (CNN) type artificial intelligences were trained to estimate the Cerenkov radiation present in the temporal response of a LINAC irradiated scintillator-fiber optic dosimeter. The CNN estimate of Cerenkov radiation is subtracted from the combined scintillation and Cerenkov radiation temporal response of the irradiated scintillator-fiber optic dosimeter, giving the sole scintillation signal, which is proportional to the scintillator dose. The CNN measured scintillator dose was compared to the background subtraction measured scintillator dose and ionisation chamber measured dose. The dose discrepancy of the CNN measured dose was on average $1.4 \%$ with respect to the ionisation chamber measured dose, matching the $1.4 \%$ average dose discrepancy of the background subtraction measured dose with respect to the ionisation chamber measured dose. The developed CNNs had an average time of $3 \mathrm{~ms}$ to calculate scintillator dose, permitting the CNNs presented to be applicable for dosimetry in real time.
\end{abstract}

Keywords: Artificial Intelligence, Megavoltage X-ray Beam, Scintillation Dosimetry, Fiber Optic Dosimeters

\footnotetext{
* Corresponding author

URL: 1jm898@uowmail.edu.au (Levi Madden)
}

Preprint submitted to Phys. Med. Biol. 


\section{Introduction}

Plastic scintillator-fiber optic dosimeters possess a unique set of qualities that make them promising in megavoltage photon beam dosimetry [1, 2]. Their desirable qualities include water equivalence across the megavoltage photon therapy energy range, dose rate independence and linear response with dose [1, 2, A typical scintillator-fiber optic dosimeter consists of a scintillator volume optically coupled to an optical fiber, where the irradiation of an optical fiber at megavoltage photon energies results in the generation of Cerenkov radiation. Cerenkov radiation is produced by an electron travelling faster than the local speed of light in an optical medium [3]; for polymethyl methacrylate (a typical optical fiber core material) the minimum photon energy that allows for Cerenkov radiation production is $320 \mathrm{keV}$ 4. In the case that a scintillator volume is not coupled to the optical fiber, only Cerenkov radiation is produced by the fiber optic dosimeter; these dosimeters are referred to as Cerenkov fiberoptic dosimeters. The application of Cerenkov fiber-optic dosimeters provides a viable alternative to scintillation dosimeters. A drawback in the application of Cerenkov fiber-optic dosimeters is a directionally dependent response with respect to irradiation angle.

The optical signal produced by a megavoltage beam irradiated scintillatorfiber optic dosimeter consists of both scintillation and Cerenkov radiation, with the two responses being independent of one another. A method that estimates or measures the Cerenkov radiation response and allows for the separation of scintillation and Cerenkov radiation is necessary for scintillation dosimetry. The scintillation response is determined by subtracting the measured Cerenkov radiation response from the measured combined scintillation and Cerenkov radiation response of the scintillator probe. The combined scintillation and Cerenkov radiation response of the scintillator probe will be referred to as the total response for simplicity.

The gold standard of Cerenkov radiation estimation methods, background subtraction, employs two fiber optic probes to measure the separated scintil- 
lation and Cerenkov radiation responses [1, 2]. The first probe, referred to as the scintillator probe, consists of a scintillator volume optically coupled to an optical fiber. The second probe, referred to as the reference probe, consists of an identical optical fiber with no scintillator volume coupled. Two identical photodetectors are used in background subtraction: one photodetector to measure the total response of the scintillator probe and the other to measure the Cerenkov radiation response of the reference probe. The reference probe is aligned with and placed beneath the scintillator probe with the aim of having equal lengths of each optical fiber irradiated. With equal lengths of fiber irradiated, the Cerenkov radiation response of the reference probe is approximately equal to the Cerenkov radiation response produced in the scintillator probe. This assumption holds true in fields with low spatial dose gradients, resulting in the background subtraction method having an average relative dose discrepancy of $0.52 \%$ [ 5 . Background subtraction is most commonly employed in the measurement of depth dose profiles and beam profiles [6]. For steep spatial dose gradient fields, the assumption is not valid due to the potential for significantly varying doses being delivered between the two probes. Other Cerenkov radiation measurement techniques are required for dosimetry in non reference conditions [5]; background subtraction is seldom employed in these conditions as the uncertainties in the background subtraction become unacceptable.

Single probe methods that employ other Cerenkov estimation techniques overcome the steep dose gradient field constraint inherent to background subtraction. For pulsed radiation sources such as clinical linear accelerators (LINACs), scintillation and Cerenkov radiation responses can be temporally separated. In these temporal methods, scintillators with decay constants in the order of hundreds of nanoseconds are chosen for separation from Cerenkov radiation, whose decay constant is in the order of nanoseconds 4]. Pulse gating methods such as temporal filtration remove $99 \%$ of the Cerenkov radiation response at the expense of a $44 \%$ loss in scintillation response. Temporal analytic methods do not experience the loss in scintillation siganl inherent to temporal filtration, however accuracy in Cerenkov radiation estimation has not yet been sufficient 
to result in clinically acceptable dose discrepancies in scintillator dose measurement [7, 8, 9, 10].

In previous work [11, shallow artificial neural networks (ANNs) were trained to estimate the Cerenkov radiation response present in measured total response waveforms. The ANN Cerenkov estimation method is a single probe method, where the ANN measured scintillator dose is calculated by subtracting Cerenkov radiation estimated by the ANN from the measured total response generated by the scintillator probe. The trained ANNs achieved an average relative dose discrepancy of $2.2 \%$ in measured scintillator dose. It was concluded that the average dose discrepancy of the ANN measured dose would be improved to levels approaching the benchmark of background subtraction by applying a deeper, more suitable neural network. In recent years, convolutional neural networks (CNNs) such as AlexNet [12, ZFnet [13, VGGnet [14, GoogleNet [15] and ResNet [16] have achieved state of the art levels of performance in image classification, with shallow ANNs unable to match this level of performance.

Convolutional neural networks were developed for the estimation of Cerenkov radiation in measured scintillator probe waveforms. The trained CNNs predict the Cerenkov radiation response in an input total response waveform that would be measured using a corresponding reference probe. Single probe scintillatorfiber optic dosimeters have been demonstrated to be clinically viable small field dosimeters [17. The CNN dose measurement method developed utilises a single probe geometry, making the presented method viable in small fields where background subtraction is unviable. The goal of this study was to improve upon the $2.2 \%$ dose discrepancy of previous work through the development of a deep CNN.

\section{Materials and Methods}

The scintillator probe consists of a cylindrical plastic scintillator (BC444 by Saint Gobain) volume with a diameter of $2 \mathrm{~mm}$ and a length of $0.5 \mathrm{~mm}$, coupled to an optical fiber (Eska CK40) with an inner core diameter of $1 \mathrm{~mm}$. The 
reference probe was constructed from an identical optical fiber (Eska CK40), with dimensions matching the fiber of the scintillator probe. Each fiber probe was coated with diffuse reflective optical paint (BC620 by Saint Gobain), increasing the scintillation collection efficiency of the scintillator probe by $30 \%$ [18. The paint coating additionally prevented background light from entering each probe at angles that would result in collection. Two matching photomultiplier tubes (RCA 4526) were used for simultaneous measurement of the total response and Cerenkov radiation responses generated in the scintillator and reference probe, respectively. A reference probe was used in this study to measure the known Cerenkov radiation responses for a given total response waveform, which are used in the training of the CNN. The photomultiplier tubes were cross calibrated to ensure the equivalence of identical responses between the two photomultiplier tubes.

A Picoscope PS6404 digital oscilloscope was used to simultaneously record the total response and Cerenkov radiation waveforms produced by the scintillator and reference probe, respectively. The transistor-transistor logic sync pulse of the LINAC (Varian 21iX Clinac) triggered the oscilloscope, enabling reproducible measurement of the two signals. A waveform recorded by the oscilloscope was the average of 100 waveforms delivered in order to decrease the noise associated with random measurement fluctuation. Waveforms recorded by the oscilloscope consisted of 10000 samples, with a sampling frequency of 625 MHz. The treatment pulse had an approximate duration of $4 \mu \mathrm{s}$, encapsulated by the $16 \mu$ s duration of each captured waveform. Measured waveforms were inverted due to polarity of the photomultiplier tube and offset due to the measurement of background signals. For the synthesis of training data, the inversion and offset were corrected with background signals having their mean response aligned with zero.

Solid water (Gammex RMI 457) was used as the phantom material for dose profile measurements at photon beam energies of $6 \mathrm{MV}$ and $10 \mathrm{MV}$. A $1 \mathrm{~cm}$ thick sheet of perpsex with a machined groove was used to house the scintillator and reference probe. $10 \mathrm{~cm}$ of solid water was placed underneath the perspex housing 
to simulate the scattering that occurs in patients as treatment is delivered. The setup is shown in Figures 1 (a) and (b). For the 6 MV energy, dose profiles were measured at the depth of maximum dose $(1.5 \mathrm{~cm})$ for field sizes of $3 \mathrm{~cm} \times 3$ $\mathrm{cm}, 5 \mathrm{~cm} \times 5 \mathrm{~cm}$ and $10 \mathrm{~cm} \times 10 \mathrm{~cm}$. For the $10 \mathrm{MV}$ energy, dose profiles were measured at the corresponding depth of maximum dose $(2.1 \mathrm{~cm})$ for a field size of $5 \mathrm{~cm} \times 5 \mathrm{~cm}$. All measurements were taken at a dose rate of $600 \mathrm{MU} / \mathrm{min}$, with a source to surface distance of $100 \mathrm{~cm}$. A Scanditronix CC13 ionisation chamber was used to measure the beam profiles for comparison with the CNN measured and background subtraction measured dose profiles.

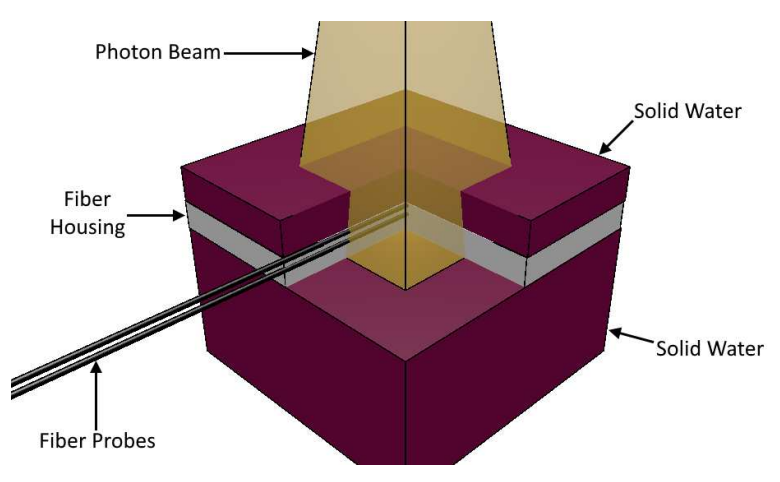

(a)

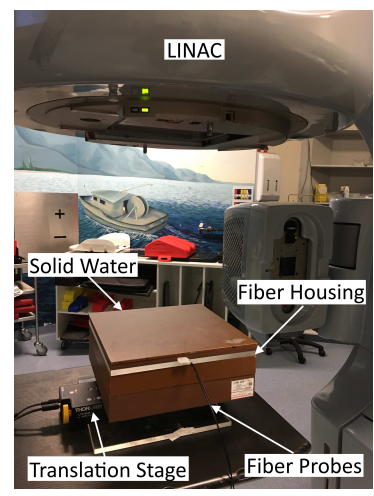

(b)

Figure 1: 3D model of the setup in solid water with a cutaway to show the probes positioning in the fiber housing (a) and picture of the experimental setup (b).

The solid water backscatter was placed on a linear translation stage (Thorlabs LTS-150). Translation of the stage allowed the movement of the fiber probes with respect to the LINACs photon beam. The translation stage was used to control the scintillator and reference probe positions for the measurement of the dose profiles. Two orientations of the fiber probes were tested during collection of the dose profiles: constant fiber length and changing fiber length orientation.

For dose profile measurement in the constant fiber length orientation, the scanning direction of the beam profile is perpendicular to the central axis of the fiber probe, with the length of the fiber irradiated remaining constant. The resultant Cerenkov radiation beam profile follows the same distribution that 
Figure 2: Cerenkov radiation response profiles for the $10 \mathrm{~cm} \times 10 \mathrm{~cm}$ field size. Position is the position of the tip of the reference probe and Response Relative to COF Scintillator is the Cerenkov radiation dose response with respect to the scintillators dose response at the centre of the $10 \mathrm{~cm} \times 10 \mathrm{~cm}$ field. Changing Fiber Length is the Cerenkov radiation response for the changing fiber length orientation and Constant Fiber Length is the Cerenkov radiation response for the constant fiber length orientation.

is measured by the scintillator volume and ionisation chamber, as in Figure 2 (blue). In the changing fiber length orientation, the scanning direction of the beam profile is parallel to the central axis of the fiber probe, with the length of fiber irradiated changing as the probes position changes. The resultant Cerenkov radiation response profile does not follow the beam profile distribution measured by the scintillator volume and ionisation chamber, as in Figure 2 (orange). The fiber probes were constrained to remain straight in the scintillator probe housing. The irradiation angle of the fiber probe for these orientations was $90^{\circ}$, optimising the trained $\mathrm{CNNs}$ performance by minimising the Cerenkov radiation collected by the optical fiber. The changing fiber length and constant fiber length orientations arise as a result of the fiber probes housing constraint.

\subsection{Convolutional Neural Network Methods}

Convolutional neural networks were developed for the measurement of Cerenkov radiation at a single beam energy as the shape of measured Cerenkov radiation responses varied with beam energy as seen in Figures 4 (a) and (b). The deep 
convolutional neural networks were developed to estimate the Cerenkov radiation response present in an input measured total response produced in the scintillator probe. To train the CNNs to correctly estimate the Cerenkov radiation response, training data must consist of the known Cerenkov radiation responses for a given corresponding total response waveform. After CNN training has been completed, the trained CNNs have learned to predict the Cerenkov radiation response that would be measured by a corresponding reference probe as in background subtraction.

The CNN architecture is modelled around the architecture of AlexNet and VGGNet [12, 14, where their then state of the art performance was achieved with a simple, deep structure. The CNNs were constructed in MATLAB (2018 a) using the Neural Network Toolbox. The CNNs structures were optimised for its performance by varying the types of layers used, order of these layers, layer size and network depth. As the CNNs structure was optimised, the dropout parameter and convolutional layers filter size was varied for further optimisation of the CNNs. The optimal structure was found to be as presented in Figure 3, differing from the AlexNet and VGGNet structures. At $6 \mathrm{MV}$, the optimally performing CNN had a dropout parameter of 0.15 and the optimal filter size was $3 \times 1$. At $10 \mathrm{MV}$, the optimally performing $\mathrm{CNN}$ had a dropout parameter of 0.1 and the optimal filter size was $3 \times 1$.

The first layer of the CNN is an average pooling layer that acts as a moving average filter with a span of 21 , serving to reduce measurement noise and improving CNN robustness with respect to signal noise. Convolutional filters in the convolutional layers had a size of $3 \times 1$ and a stride of 1 . Subsequent batch normalisation [19] and leaky ReLU layers were used as the activations in the CNN, with 0.1 as the leaky ReLU parameter. The average pooling layers had a size of $2 \times 1$ and a step size of 2 , reducing an input $\mathrm{N}$ x 1 array to an $(\mathrm{N} / 2) \mathrm{x}$ 1 array. Dropout layers were implemented to improve CNN performance [20]. 
Figure 3: Architecture of the CNN developed. The notation $3 \times 1$ conv 4 at layer 2 denotes that four $3 \times 1$ convolutions are independently applied to the incoming $3500 \times 1$ array, with the output being a $3500 \times 1 \times 4$ array. The average pooling layers at layers 5, 9, 13, 17 and 21 downsample the incoming waveforms by a factor of 2 . The activation parameter for each leaky ReLU layer was 0.1. The dropout rate for each dropout layer was 0.15 for the $6 \mathrm{MV}$ energy and 0.1 for the $10 \mathrm{MV}$ energy.

\subsubsection{Waveform Preprocessing}

The Cerenkov radiation responses of the reference probe and total responses of the scintillator probe were measured simultaneously by the oscilloscope. The waveforms recorded by the oscilloscope were 10000 elements long, for a waveform duration of $16 \mu \mathrm{s}$. Approximately $5.5 \mu \mathrm{s}$ of the $16 \mu \mathrm{s}$ waveform duration were comprised of measurable scintillation, with the remaining $10.5 \mu$ s comprised solely of background signals as in Figure 4 (a). Recorded waveforms were sampled about the measurable scintillation, as in Figure 4 (b), eliminating the temporal regions comprised solely of background signals. These measured waveforms were shortened to a length and duration of 3500 elements and 5.6 $\mu \mathrm{s}$, respectively. The shortening of waveforms decreased the time required to train each $\mathrm{CNN}$. 


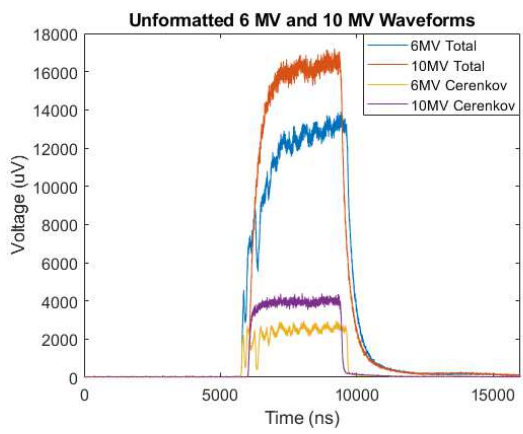

(a)

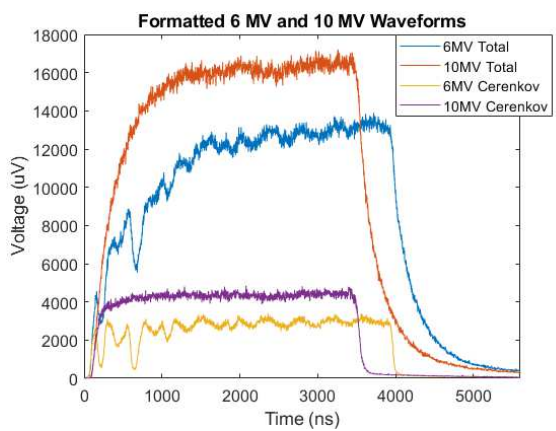

(b)

Figure 4: Total response and Cerenkov radiation response waveforms prior to processing (a) and post processing (b) as generated in the scintillator and reference probes, respectively. $6 \mathrm{MV}$ Total and $10 \mathrm{MV}$ Total are the total response signals generated in the scintillator probe at the $6 \mathrm{MV}$ and $10 \mathrm{MV}$ beam energies, respectively. 6MV Cerenkov and 10MV Cerenkov is the Cerenkov radiation generated in the reference probe at the $6 \mathrm{MV}$ and $10 \mathrm{MV}$ energies, respectively.

\subsubsection{Synthesis of Training Data}

Deep CNNs require many training samples to reach the high accuracies that are superior to simpler artificial neural networks. For the CNNs developed in the work presented, it was expected that the number of training samples required was of the order of tens of thousands. Instead of experimentally recording tens of thousands of waveforms for training, training data was generated synthetically from a smaller set of recorded data. The experimentally recorded data was not included in the training data and instead used as testing data. Experimentally measured data was separated into subsets according to beam energy. For each subset of measured data, training data was synthesised following the steps below:

1. Perform background subtraction to determine the sole scintillation response for a corresponding Cerenkov radiation response.

2. For each sole scintillation and Cerenkov radiation response, record the minimum and maximum signal levels, as well as the amplitude (defined as the difference between the maximum and minimum signal levels of a sole response). Determine the global minimum and maximum signal levels 
for the set of sole scintillation responses; repeat for the set of Cerenkov radiation responses. Record the maximum and minimum amplitudes of scintillation and Cerenkov radiation signals.

3. Apply min-max normalisation to each sole scintillation response using the global minimum and maximum signal levels of scintillation. Repeat for each Cerenkov radiation response using the global minimum and maximum signal levels of Cerenkov radiation. These normalised responses serve as models for the expected Cerenkov radiation and scintillation responses.

4. Scintillation and Cerenkov radiation dose response profiles are modelled for the scintillator probe in the changing fiber length orientation. An empirical model is used to approximate the scintillation dose response profile, as in Equation 1. The Cerenkov radiation response profile is the theoretical response of the optical fiber to the photon beam whose profile is equivalent to the scintillator response profile, as in Equation 2:

$$
S(x, F S)=\frac{S_{\min }}{S_{\min }+S_{\max }}+\frac{S_{\max }}{S_{\min }+S_{\max }} \cdot \frac{1}{1+\exp (-4 \sqrt{2} \cdot|x+0.5 F S|)}
$$

$$
C(x, F S)=\frac{C_{\min }}{C_{\min }+C_{\max }}+\frac{C_{\max }}{C_{\min }+C_{\max }} \cdot \int_{-\infty}^{x} \frac{S\left(x^{\prime}, F S\right) d x^{\prime}}{F S_{r e l}}
$$

$S(x, F S)$ and $C(x, F S)$ are the relative dose responses of scintillation and Cerenkov radiation as a function of probe position, $x$, and the width of the modelled photon beam, FS. $S_{\min }$ and $S_{\max }$ are the minimum and maximum scintillation signal amplitudes, while $C_{\min }$ and $C_{\max }$ are the minimum and maximum Cerenkov radiation signal amplitudes. $F S_{r e l}$ is the maximum width of experimentally measured beam profiles, compensating for the linear relationship between the length of optical fiber irradiated and the magnitude of measured Cerenkov radiation responses 44. The constant $4 \sqrt{2}$ in Equation 1 is added to make sure all the dose profiles generated will have a constant penumbra width of $5 \mathrm{~mm}$, which is 
the known penumbra width for this LINAC configuration [11]. The mod-

elled dose profiles for the $6 \mathrm{MV}, 5 \mathrm{~cm} \times 5 \mathrm{~cm}$ and $10 \mathrm{~cm} \times 10 \mathrm{~cm}$ field sizes are plotted against the measured $6 \mathrm{MV}, 5 \mathrm{~cm} \times 5 \mathrm{~cm}$ and $10 \mathrm{~cm} \times$ $10 \mathrm{~cm}$ dose profiles in Figures 5 (a) and 5 (b).

5. Dose profiles are modelled for $F S=(1,1.2,1.4, \ldots, 14.6,14.8,15) \mathrm{cm}$. The modelled profiles are sampled for their relative dose across the positions $x=(-1.5 F S,-1.48 F S,-1.46 F S, \ldots, 1.46 F S, 1.48 F S, 1.5 F S)$.

6. A random pair of normalised sole scintillation and Cerenkov radiation responses (as in step 3) are chosen for each combination of position and modelled photon beam width. The random pair of sole responses are scaled by their respective relative doses (as in step 4) and their respective maximum signal amplitude (as in step 2). These resultant scaled responses are the sole scintillation and Cerenkov radiation responses that are expected to be produced experimentally.

7. A training input waveform is synthesised by adding the sole synthetic scintillation response, the sole synthetic Cerenkov radiation response and Gaussian noise. A training Cerenkov radiation waveform is synthesised by adding zero mean Gaussian noise to the sole synthetic Cerenkov radiation response. The training set is developed by synthesising training waveforms across the 10721 combinations of $F S$ and $x$.

CNNs were also developed to measure the scintillation response present in input waveform, however, the optimally performing CNNs were trained to measure Cerenkov radiation instead of scintillation. The presented method for data synthesis can be applied with the final step modified to produce target waveforms comprised solely of scintillation.

\section{Results}

For the $6 \mathrm{MV}, 5 \mathrm{~cm} \times 5 \mathrm{~cm}$ dose profile presented in Figure 6 , the $\mathrm{CNN}$ measured scintillator dose was underestimated with respect to the background 


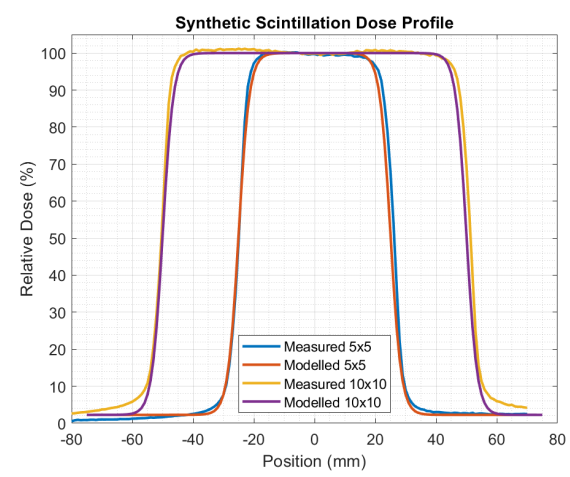

(a)

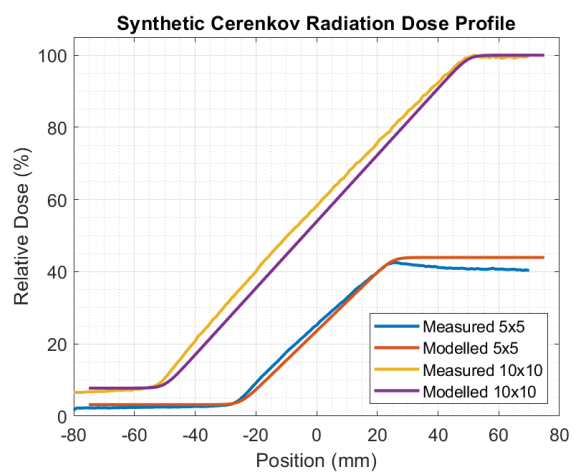

(b)

Figure 5: (a) Experimentally measured scintillator and modelled scintillator dose profiles and (b) the corresponding experimentally measured and modelled Cerenkov radiation dose profile in the changing fiber length orientation at $6 \mathrm{MV}$. Measured 5x5 and Measured 10x10 responses are the experimentally measured responses for the $5 \mathrm{~cm} \times 5 \mathrm{~cm}$ and $10 \mathrm{~cm} \times 10 \mathrm{~cm}$ fields. Modelled 5x5 and Modelled 10x10 are the modelled $5 \mathrm{~cm} \times 5 \mathrm{~cm}$ and $10 \mathrm{~cm} \times 10 \mathrm{~cm}$ dose responses used in the generation of synthetic training data. In (a), Relative Dose is the dose relative to the centre of field dose. However, in (b), Relative Dose is the dose relative to the maximum dose response of the measured $10 \mathrm{~cm} \times 10 \mathrm{~cm}$ profile.

subtraction measured dose for out of field positions. A tilt is present in the central region of the CNN measured dose profile due to incorrect estimation of the Cerenkov radiation present in the total response waveforms. The background subtraction measured dose has an asymmetry such that the dose is overestimated for positions between $20 \mathrm{~mm}$ and $70 \mathrm{~mm}$, increasing the average dose discrepancy of background subtraction method with respect to the ionisation chamber. The average dose discrepancy in CNN measured scintillator dose and background subtraction measured scintillator dose with respect to the ionisation chamber measured dose are presented in Table 1.

The CNN measured scintillator dose closely matches the background subtraction measured scintillator dose and ionisation chamber measured dose for the $6 \mathrm{MV}, 10 \mathrm{~cm} \times 10 \mathrm{~cm}$ dose profiles presented in Figures 7 and 8 . The average dose discrepancy of the $\mathrm{CNN}$ measured scintillator dose matched and improved upon the average dose discrepancy of the background subtraction measured 


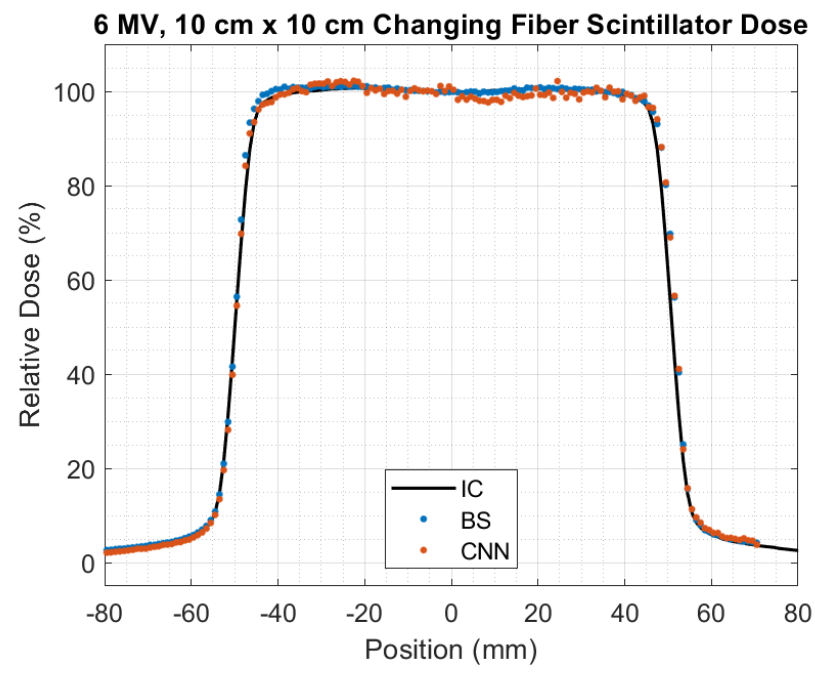

Figure 7: Scintillator dose profile for a $10 \mathrm{~cm} \times 10 \mathrm{~cm}$ field in the changing fiber length orientation. IC, BS and $\mathrm{CNN}$ are as defined in Figure 6. 
scintillator dose, as presented in Table 1 . For the $10 \mathrm{~cm} \times 10 \mathrm{~cm}$ field size, the scintillator measured dose profile penumbras are narrower and steeper than the ionisation chamber measured dose profile penumbras, arising from the smaller sensitive volume and improved resolution of the scintillator probe compared to the ionisation chamber.

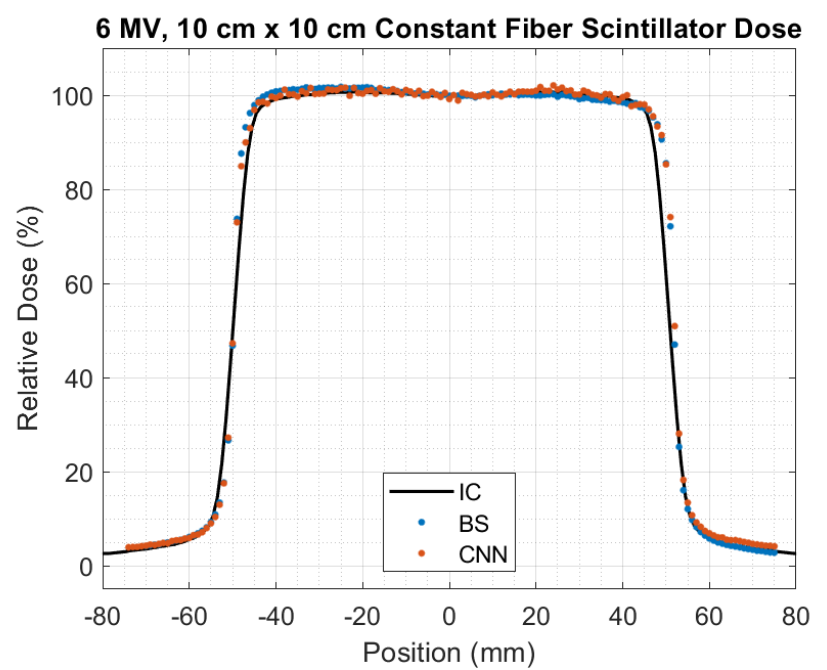

Figure 8: Scintillator dose profile for a $10 \mathrm{~cm} \times 10 \mathrm{~cm}$ field in the constant fiber length orientation. IC, BS and $\mathrm{CNN}$ are as defined in Figure 6.

The $6 \mathrm{MV}$ CNN was applied for dose measurements on testing data that was not used to generate the synthetic training data. A $6 \mathrm{MV}, 3 \mathrm{~cm} \times 3 \mathrm{~cm}$ dose profile was measured at a depth of $1.5 \mathrm{~cm}$ and a SSD of $100 \mathrm{~cm}$. The 3 $\mathrm{cm} \times 3 \mathrm{~cm}$ dose profile was also measured using background subtraction and an ionisation chamber. The $3 \mathrm{~cm} \times 3 \mathrm{~cm}$ dose profile is plotted in Figure 9 . The dose discrepancies were $3.4 \%$ and $4.0 \%$ for background subtraction and the CNN with respect to the ionisation chamber. The increased dose discrepancies of the $3 \mathrm{~cm} \times 3 \mathrm{~cm}$ dose profiles arise due to the mismatch in penumbra widths when comparing the scintillator measured and ionisation chamber measured dose profiles.

The $10 \mathrm{MV}$ CNN was applied for dose measurements on the $10 \mathrm{MV}, 5 \mathrm{~cm} \times$ 
Figure 9: Scintillator dose profile for a $3 \mathrm{~cm} \times 3 \mathrm{~cm}$ field in the constant fiber length orientation. IC, BS and CNN are as defined in Figure 6.

$5 \mathrm{~cm}$ dose profile. The $10 \mathrm{MV}, 5 \mathrm{~cm} \times 5 \mathrm{~cm}$ dose profile was also measured using background subtraction and an ionisation chamber, shown plotted in Figure 10. The CNN measured dose is overestimated at the positions between $12 \mathrm{~mm}$ and $21 \mathrm{~mm}$ attributed to incorrect estimation of the measured dose.

The average dose discrepancies in Table 1 were calculated with respect to the ionisation chamber measured dose. For the dose discrepancies presented in Table 1, the average dose discrepancy of the CNN measured dose was $1.4 \%$, matching the average dose discrepancy of the background subtraction measured scintillator dose of $1.4 \%$. The mismatch in penumbra widths between the ionisation chamber and scintillator profiles (Figure 9) inflated the dose discrepancies for the $6 \mathrm{MV}, 3 \mathrm{~cm} \times 3 \mathrm{~cm}$ field, and so the $6 \mathrm{MV}, 3 \mathrm{~cm} \times 3 \mathrm{~cm}$ dose discrepancies were excluded when calculating the mean dose discrepancies in Table 1. 


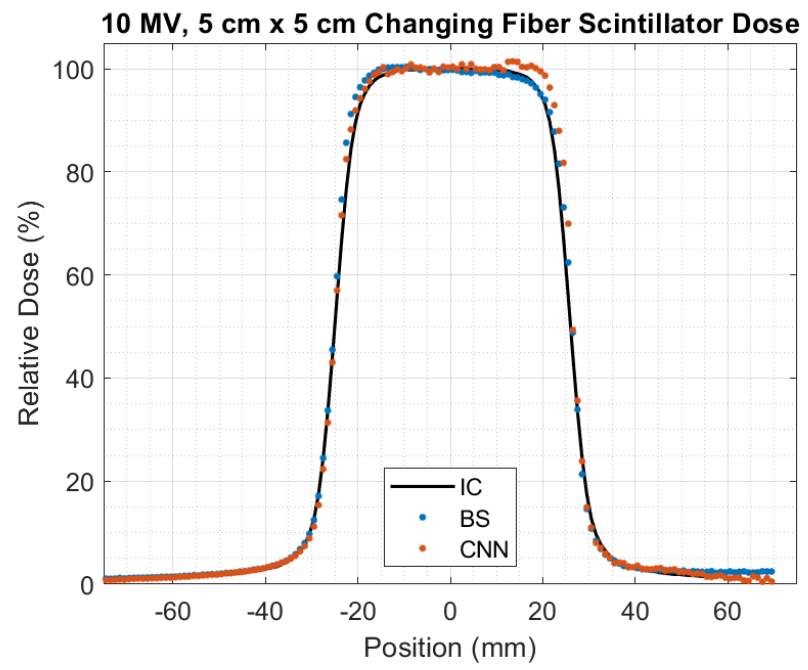

Figure 10: Scintillator dose profile for a $10 \mathrm{MV}, 5 \mathrm{~cm} \times 5 \mathrm{~cm}$ field in the changing fiber length orientation. IC, BS and CNN are as defined in Figure 6.

\begin{tabular}{|c|c|c|c|}
\hline $\begin{array}{c}\text { Energy } \\
(\mathrm{MV})\end{array}$ & $\begin{array}{c}\text { Field size } \\
\left(\mathrm{cm}^{2}\right)\end{array}$ & $\begin{array}{c}\text { CNN dose } \\
\text { discrepancy (\%) }\end{array}$ & $\begin{array}{c}\text { BS dose } \\
\text { discrepancy }(\%)\end{array}$ \\
\hline 6 & $5 \times 5$ & 1.6 & 1.4 \\
6 & $10 \times 10$ & 1.2 & 1.4 \\
6 & $10 \times 10^{*}$ & 1.5 & 1.6 \\
10 & $5 \times 5$ & 1.4 & 1.1 \\
Mean & - & 1.4 & 1.4 \\
\hline
\end{tabular}

Table 1: Field sizes: $5 \times 5$ is the $5 \mathrm{~cm} \times 5 \mathrm{~cm}$ field for the changing fiber length orientation, $10 \times 10$ is the $10 \mathrm{~cm} \times 10 \mathrm{~cm}$ field for the changing fiber length orientation and $10 \times 10^{*}$ is the $10 \mathrm{~cm} \times 10 \mathrm{~cm}$ field for the constant fiber length orientation. Mean is the mean value of the dose discrepancies listed for the $6 \mathrm{MV}$ and $10 \mathrm{MV}$ beam energies, excluding the $6 \mathrm{MV}, 3 \mathrm{~cm} \times$ $3 \mathrm{~cm}$ discrepancies. CNN dose discrepancy is the average dose discrepancy between the CNN measured scintillator dose and ionisation chamber dose at corresponding positions. BS dose discrepancy is the average dose discrepancy between the background subtraction measured scintillator dose and ionisation chamber dose at corresponding positions.

\section{Discussion}

The resolution of the scintillator probe was $2 \mathrm{~mm}$ for the changing fiber length orientation (arising from the scintillator diameter) and $0.5 \mathrm{~mm}$ for the 
constant fiber length dose profile (arising from the scintillator length), while the resolution of the ionisation chamber was $6 \mathrm{~mm}$. The resultant dose profiles measured by the scintillator probe have penumbras that are narrower and steeper than the penumbras measured with the ionisation chamber, as in Figures 6-10. The improvement in resolution with the scintillator probe leads to an increase in the scintillator measured dose discrepancies calculated with respect to the ionisation chamber.

The Cerenkov radiation generated in our system reaches a maximum response of approximately $54 \%$ for the $10 \mathrm{~cm} \times 10 \mathrm{~cm}$ field relative to the centre of field scintillator response, as shown in Figure 2 (b). The scintillator position where this responses occurs for the $10 \mathrm{~cm} \times 10 \mathrm{~cm}$ changing fiber length dose profile is at $70 \mathrm{~mm}$ (Figure 7), where the scintillator response is approximately $8 \%$ relative to the centre of field scintillator response. The total response at this point is comprised of approximately $87 \%$ Cerenkov radiation and $13 \%$ scintillation. The CNN measured scintillator dose closely matched the ionisation chamber measured dose at this position where Cerenkov radiation response dominates the total response. Conversely, the CNN measured scintillator dose closely matched the ionisation chamber measured dose at positions where Cerenkov radiation responses were minimal, corresponding to positions about the $-80 \mathrm{~mm}$ position in Figure 7. The close agreement of the CNN measured scintillator dose with respect to the ionisation chamber measured doses in Figures 6 and 7 demonstrate the effectiveness of the CNN across the experimentally measured range of scintillation and Cerenkov radiation responses.

A CNN trained on synthetic data achieves its optimal performance when the synthetic training data is generated from a model capable of exactly reproducing measured data 21]. The dose profile models (Equations 1 and 2) are empirical models rather than theoretically derived models. For the $10 \mathrm{~cm} \times 10 \mathrm{~cm}$ field, the modelled scintillation beam profiles were on average within $2.2 \%$ of the measured relative dose, with a maximum deviation of $29 \%$ as in Figures 5 (a). For the same $10 \mathrm{~cm} \times 10 \mathrm{~cm}$ field, the modelled Cerenkov radiation beam profiles were on average within $2.0 \%$ of the measured Cerenkov radiation response, with 
a maximum deviation of $4.1 \%$ as in Figure 5 (b). For the corresponding $5 \mathrm{~cm}$ $\times 5 \mathrm{~cm}$ dose profiles, the modelled scintillator dose and Cerenkov radiation response deviated by average relative doses of $3.0 \%$ and $2.6 \%$, respectively.

The scintillator volume produces additional Cerenkov radiation that was unaccounted for in its corresponding model (Equation 2), leading to increased deviations as field size decreased. As the training data was synthesised from sole scintillation and sole Cerenkov radiation signals, the magnitude of Cerenkov radiation in training input waveforms matches the magnitude present in target waveforms. The CNN has been trained to measure all Cerenkov radiation present in an input waveform, thus the CNN implicitly accounts for Cerenkov radiation generated in the scintillator volume. Optimising CNN performance by improving the synthetic data generation model and improving dose reproducibility will be the aim of future work.

With variations in the LINACs waveform, CNN performance degradation is anticipated. Cerenkov radiation has an intensity proportional to the LINACs dose rate 22]; the shape of temporal Cerenkov radiation responses follow the shape of the LINACs treatment pulse waveforms. CNNs developed for Cerenkov radiation measurement are applicable only for the LINAC waveform used during the collection of training data. For LINACs where their waveforms remain constant as a function of beam energy, CNNs developed and trained following the presented method are applicable at other beam energies as plastic scintillation dosimeters responses are energy independent for LINAC beams [2].

A trained CNNs performance is anticipated to degrade as magnitudes of scintillation and Cerenkov radiation exceed their maximum magnitudes in the training set 23. Similar performance degradation is expected as measured magnitudes fall below their minimum magnitudes in the training set [23]. The intensities of Cerenkov radiation and scintillation are linear with respect to dose [1, 22]; variations of depth and SSD results in variations of the magnitudes of scintillation and Cerenkov radiation produced. Variations in the optical fiber size, scintillator size and irradiation angle result in variations in the magnitudes of scintillation and Cerenkov radiation measured. The trained CNNs 
performance remains undegraded for variations of these factors, provided that measured magnitudes of scintillation and Cerenkov radiation remain between the minimum and maximum amplitudes of each response in the training set.

Once a CNN has been trained, the CNN can be applied for dose measurement with no calibration measurements required. The CNN developed had an average dose discrepancy in its scintillator dose matching the background subtraction measured scintillator dose. The CNN Cerenkov radiation estimation methods ability to perform single probe scintillator dose measurements with no calibration measurements and average dose discrepancy of background subtraction highlights the promise of the presented method. Future work will aim at applying the CNN method for small field dosimetry with clinical accuracy.

The time required to train a CNN was on average 4 minutes using a medium range laptop. The LINAC applied delivered pulses at a frequency of $360 \mathrm{~Hz}$; with 100 pulses averaged per recorded waveform, the minimum time required to record a single waveform is $28 \mathrm{~ms}$. The time taken for a network to predict the expected Cerenkov radiation from an input total response waveform was 3 $\mathrm{ms}$, rendering the CNNs trained capable of real time dosimetry.

\section{Conclusion}

The CNN measured scintillator dose had an average dose discrepancy matching the average dose discrepancy of the background subtraction dose; dose discrepancies were calculated with respect to the relative dose measured by an ionisation chamber. These dose discrepancies (background subtraction and CNN Cerenkov estimation) were on average $1.4 \%$. Once CNNs have been trained, the CNN method of Cerenkov radiation estimation achieved a level of performance matching background subtraction. A reference probe is required for the measurement of known Cerenkov radiation response waveforms to train the CNNs. The reference probe is not required by the presented CNN method for scintillator dose calculations after the training is complete. The CNN method of Cerenkov radiation estimation requires only the scintillator probe after train- 
ing, where background subtraction requires the additional reference probe. The CNN Cerenkov estimation method is capable of being employed for real time dosimetry, with the time required for the $\mathrm{CNN}$ to process an input waveform being $3 \mathrm{~ms}$.

\section{Acknowledgements}

This project was supported by UOWs Global Challenges Program. This research has been conducted with the support of the Australian Government Research Training Program Scholarship.

[1] A. S. Beddar, T. R. Mackie, F. H. Attix, Water-equivalent plastic scintillation detectors for high-energy beam dosimetry .1: physical characteristics and theoretical considerations, Physics in Medicine and Biology 37 (1992) $1883-1900$

[2] A. S. Beddar, T. R. Mackie, F. H. Attix, Water-equivalent plastic scintillation detectors for high-energy beam dosimetry .2: properties and measurements, Physics in Medicine and Biology 37 (1992) 1901-1913.

[3] P. A. Cerenkov, Visible radiation produced by electrons moving in a medium with velocities exceeding that of light, Physical Review 52 (1937) 378-379.

[4] K. W. Jang, T. Yagi, C. H. Pyeon, W. J. Yoo, S. H. Shin, C. Jeong, B. J. Min, D. Shin, T. Misawa, B. Lee, Application of cerenkov radiation generated in plastic optical fibers for therapeutic photon beam dosimetry, Journal of Biomedical Optics 18 (2013) 1-7.

[5] L. Archambault, A. S. Beddar, L. Gingras, R. Roy, L. Beaulieu, Measurement accuracy and Cerenkov removal for high performance, high spatial resolution scintillation dosimetry, Medical Physics 33 (1) (2006) 128-135.

[6] G. Bartesaghi, V. Conti, D. Bolognini, S. Grigioni, V. Mascagna, M. Prest, S. Scazzi, A. Mozzanica, P. Cappelletti, M. Frigerio, S. Gelosa, A. Monti, 
A. Ostinelli, G. Giannini, E. Vallazza, A scintillating fiber dosimeter for radiotherapy, Nuclear Instruments and Methods in Physics Research Section A: Accelerators, Spectrometers, Detectors and Associated Equipment 581 (1) (2007) $80-83$.

[7] J. Archer, L. Madden, E. Li, M. Carolan, M. Petasecca, P. Metcalfe, A. Rosenfeld, Temporally separating cherenkov radiation in a scintillator probe exposed to a pulsed x-ray beam, Physica Medica 42 (2017) 185 188.

[8] J. Archer, E. Li, Recent advances in photonic dosimeters for medical radiation therapy, Frontiers of Optoelectronics 11 (1) (2018) 23-29.

[9] J. Archer, L. Madden, E. Li, M. Carolan, A. Rozenfeld, A comparison of temporal cherenkov separation techniques in pulsed signal scintillator dosimetry, Biomedical Physics and Engineering Express 4.

[10] J. Archer, L. Madden, E. Li, D. Wilkinson, A. Rosenfeld, An algorithmic approach to single probe cherenkov removal in pulsed x-ray beams, Medical Physics X (2018) AAAA.

[11] L. Madden, J. Archer, E. Li, M. Carolan, A. Rosenfeld, Temporal separation of scintillation and cerenkov radiation using artificial neural networks in clinical linacs, Physica Medica 54 (2018) 131-136.

[12] A. Krizhevsky, I. Sutskever, G. E. Hinton, Imagenet classification with deep convolutional neural networks, in: F. Pereira, C. J. C. Burges, L. Bottou, K. Q. Weinberger (Eds.), Advances in Neural Information Processing Systems 25, Curran Associates, Inc., 2012, pp. 1097-1105.

[13] M. Zeiler, R. Fergus, Visualizing and understanding convolutional networks, in: Computer Vision, ECCV 2014 - 13th European Conference, Proceedings, part 1 Edition, Vol. 8689 LNCS of Lecture Notes in Computer Science (including subseries Lecture Notes in Artificial Intelligence 
and Lecture Notes in Bioinformatics), Springer Verlag, 2014, pp. 818-833. doi:10.1007/978-3-319-10590-1_53

[14] K. Simonyan, A. Zisserman, Very deep convolutional networks for largescale image recognition (2014).

[15] C. Szegedy, W. Liu, Y. Jia, P. Sermanet, S. Reed, D. Anguelov, D. Erhan, V. Vanhoucke, A. Rabinovich, Going deeper with convolutions (2014).

[16] K. He, X. Zhang, S. Ren, J. Sun, Deep residual learning for image recognition, 2016 IEEE Conference on Computer Vision and Pattern Recognition (CVPR) (2016) 770-778.

[17] M. Pasquino, C. Cutaia, L. Radici, S. Valzano, E. Gino, C. Cavedon, M. Stasi, Dosimetric characterization and behaviour in small x-ray fields of a microchamber and a plastic scintillator detector, The British Journal of Radiology 90 (1069) (2017) 20160596.

[18] J. Archer, E. Li, M. Petasecca, A. Stevenson, J. Livingstone, A. Dipuglia, J. Davis, A. Rosenfeld, M. Lerch, Synchrotron x-ray microbeam dosimetry with a 20micrometre resolution scintillator fibre-optic dosimeter, Journal of Synchrotron Radiation 25 (3) (2018) 826-832.

[19] S. Ioffe, C. Szegedy, Batch normalization: Accelerating deep network training by reducing internal covariate shift, in: ICML, 2015.

[20] H. Wu, X. Gu, Towards dropout training for convolutional neural networks, Neural Networks 71 (2015) $1-10$.

[21] T. Anh Le, A. G. Baydin, R. Zinkov, F. Wood, Using synthetic data to train neural networks is model-based reasoning, 2017 International Joint Conference on Neural Networks (IJCNN).

[22] Y. Helo, I. Rosenberg, D. D’Souza, L. Macdonald, R. Speller, G. Royle, A. Gibson, Imaging cerenkov emission as a quality assurance tool in electron radiotherapy, Physics in Medicine and Biology 59 (2014) 1963-1978. 
[23] R. Longadge, S. Dongre, Class imbalance problem in data mining review, Int. J. Comput. Sci. Netw. 2. 